\title{
Les Amours d'Armide de Pierre Joulet ou la Jérusalem romancée : analyse d'un discours de rupture amoureuse
}

\section{Adrienne Petit}

\section{(2) OpenEdition Journals}

Electronic version

URL: http://journals.openedition.org/rhetorique/823

DOI: $10.4000 /$ rhetorique.823

ISSN: 2270-6909

\section{Publisher}

UGA Éditions/Université Grenoble Alpes

\section{Printed version}

ISBN: 978-2-37747-066-2

\section{Electronic reference}

Adrienne Petit, «Les Amours d'Armide de Pierre Joulet ou la Jérusalem romancée : analyse d'un discours de rupture amoureuse », Exercices de rhétorique [Online], 12 | 2019, Online since 30 January 2019, connection on 12 September 2020. URL : http://journals.openedition.org/rhetorique/823 ; DOI https://doi.org/10.4000/rhetorique.823

This text was automatically generated on 12 September 2020 .

\section{c) (i) (2) (2)}

Les contenus de la revue Exercices de rhétorique sont mis à disposition selon les termes de la Licence Creative Commons Attribution - Pas d'Utilisation Commerciale - Partage dans les Mêmes Conditions 4.0 International. 


\title{
Les Amours d'Armide de Pierre Joulet ou la Jérusalem romancée : analyse d'un discours de rupture amoureuse
}

\author{
Adrienne Petit
}

1 En 1596, un an tout juste après la parution de la fameuse traduction de Blaise de Vigenère, l'éditeur Abel L'Angelier publie la première des adaptations romancées de $L a$ Jérusalem délivrée. Comme l'explique Jean Basalmo, Les Amours d'Armide de Pierre Joulet, sieur de Châtillon, est destiné à tirer profit de la fortune éditoriale du Tasse qui bénéficie alors d'un engouement extraordinaire auprès du lectorat français. Après la parution coup sur coup de trois traductions entre 1595 et $1596^{1}$, c'est au tour des réécritures de faire florès. Les Amours d'Armide, qui rencontre un très grand succès commercial, comme en attestent les quatorze émissions et éditions différentes qui paraissent jusqu'en 1614, fait bientôt des émules. Entre 1597 et 1600, l'exploitation de ce filon éditorial donne ainsi lieu à trois autres romans sentimentaux se présentant comme la réécriture ou la continuation des amours d'Armide et Renaud ou de Tancrède et Clorinde, l'autre couple phare du romanzo italien². Il s'agit de rivaliser d'éloquence avec la virtuosité stylistique du Tasse et avec la langue italienne, idiome par excellence de l'amour. Selon le couple de protagonistes choisi, les morceaux de bravoure sont constitués de la déploration de Tancrède sur la mort de Clorinde ou bien du portrait d'Armide et de sa plainte de colère contre Renaud, trois passages fameux de La Jérusalem délivrée.

2 Unique œuvre de fiction d'un auteur peu connu qui, en tant que ressortissant de la petite noblesse, a sans doute reçu une formation oratoire ${ }^{3}$, Les Amours d'Armide témoigne à la fois d'un moment particulier de l'histoire de l'art oratoire, celui du grand style des passions, et d'un moment éminemment rhétorique de la poétique romanesque. Après avoir montré comment Pierre Joulet fabrique un roman sentimental à partir d'un épisode épique, nous analyserons en détail la version qu'il propose de la plainte d'Armide. Ce discours est l'un des morceaux d'éloquence féminine pathétique les plus célèbres de l'âge classique, comme en témoignent, bien après la 
période de notre étude, la tragédie en musique de Quinault et Lully intitulé Armide (1686) ou encore l'héroïde Armide à Renaud que fait paraître Charles-Pierre Colardeau en 1758. C'est aussi l'une des plaintes les plus commentées qui représente, aux yeux des poéticiens du second XvII ${ }^{\mathrm{e}}$ siècle, le « clinquant du Tasse ${ }^{4}$ » et l'alliance néo-asianiste du pathos et des fleurs de rhétorique. Si, dans Il Predicatore (1609), le rhétoricien italien Francesco Panigarola justifie l'ornementation du discours d'Armide par son caractère artificieux ${ }^{5}$, Dominique Bouhours, lui, n'y voit plus que le stigmate de l'affectation italienne :

Les Poètes italiens ne sont guéres naturels, ils fardent tout; \& le Tasse par ce seul endroit est bien au dessous de Virgile. Quelle différence entre l'adieu de Didon à Enée \& celuy d'Armide à Renaud ? Ce que pense \& ce que dit la Reine de Carthage est une expression de l'amour le plus tendre \& le plus violent qui fut jamais ; c'est la nature elle-mesme qui la fait parler : au lieu qu'Armide ne pense \& ne dit presque rien de naturel ${ }^{6}$.

Comme l'indique Bouhours, par la voix d'Eudoxe, cette plainte, ou devrions-nous dire ces plaintes puisqu'elles sont trois à se succéder dans la bouche d'Armide, sont en effet imitées de celles que Didon adresse à Énée dans l'épopée virgilienne.

Nous restreignant à la seconde de ces plaintes, qui constitue le discours de rupture (exprobratio) en lui-même, nous analyserons la réécriture que Le Tasse propose de Virgile, par le truchement de la traduction de Vigenère, puis, de manière plus approfondie, celle que Joulet propose du Tasse. Tout en s'inscrivant dans la continuité des nombreux travaux sur Virgile déjà parus dans la revue, la mise en série de ces trois textes (Virgile, Le Tasse-Vigenère, Joulet), nous permettra d'apprécier les variations introduites par le romancier, tant sur le plan formel qu'herméneutique.

\section{La Jérusalem romancée : inflexion sentimentale et amplification rhétorique}

Dans l'épître qui ouvre Les Amours d'Armide, Pierre Joulet loue l'éloquence de sa dédicataire, Mademoiselle de Guise, et la prie de s'en servir pour défendre l'ouvrage qu'il place sous sa protection, en le lui dédiant. Au-delà des compliments d'usage, cette dédicace met au jour le défi que représente l'imitation du Tasse, qui est alors considéré comme " le " grand poète épique et comme un styliste hors pair. Néanmoins, tout en s'appuyant sur l'autorité de ce dernier, Joulet se justifie des libertés qu'il a prises avec son modèle :

gratifiez-moy en faveur du Tasso que vous lisez avec tant de plaisir; C'est de luy que j'ay pris le subject lequel j'ay reduict en Histoire continuë, au lieu qu'elle est inegallement espanduë dans tous les chants de cest Autheur. Et d'autant qu'il me semble que ce Poëte a rendu quelques passions, sinon du tout muettes; au moins un peu plus retenues que leur naturel, je leur ay laissé la voix libre, \& leur ay faict dire ce que j'ay pensé que l'on ressent au milieu du desplaisir, ou de la joye qu'elles nous apportent ${ }^{7}$.

Pour transformer l'épisode amoureux d'une épopée en un roman sentimental, il explique ainsi avoir recomposé l'histoire d'Armide et Renaud selon un ordre chronologique et avoir cherché à rendre son récit plus pathétique. Ces deux principes de réécriture semblent répondre directement à la critique que Vigenère adressait au Tasse au sujet du caractère inabouti et incomplet des épisodes amoureux, dans les annotations qui accompagnent sa traduction : 
il precipite beaucoup de choses qu'il couppe court, \& les laisse là imparfaictes \& entrerompues, comme les attentes d'un edifice ; frustrant les lecteurs du desir qu'ils auroient de les veoir un peu mieux promener \& resouldre: Ainsi que sont les amours si impetueuses de Tancred alendroit de Clorinde pour ne l'avoir qu'une fois veuë, \& encore bien abruptement ; \& jamais hanté, practiqué ne devisé avec elle, pour en tracer si tost une ainsi pitoyable \& tragique issue; dont on ne se souvient plus puis apres. Tout de mesme, de tant d'afflictions \& langueurs de ceste pauvre desolée Hermine pour l'amour d'iceluy Tancred, qu'il laisse là irresoluë, \& comme ravie en un instant en un silence perpetuel, n'en estant jamais plus memoire. Les beautez aussi d'Armide, \& ses enchantemens sont par trop legierement parcourus, apres une si grand' levée de bouclier qu'il en fait ${ }^{8}$.

Joulet respecte en effet l'unité d'action : il resserre son récit autour du couple d'Armide et Renaud, sans reprendre le découpage en chants qui structuraient le romanzo italien. Le romancier suit strictement l'ordo naturalis, en passant d'un fil narratif à l'autre, et supprime les analepses par lesquelles étaient rapportés, au chant X, l'ensorcellement des chevaliers chrétiens par Armide puis leur libération par Renaud et, au chant XIV, le coup de foudre d'Armide pour Renaud. La recherche d'un pathos débridé, qui est alors en vogue dans les romans sentimentaux, se traduit par le primat accordé à la matière amoureuse au détriment de la matière épique et historique ${ }^{9}$. Les épisodes guerriers, comme le conflit entre Renaud et Gernand (chant V), le triomphe de l'armée du roi d'Égypte, qui donnait lieu chez le Tasse à un véritable exposé géographique et moral des différents peuples d'Afrique, ou encore l'ekphrasis du bouclier de Renaud (chant XVII), qui était suivie d'une généalogie de ses ancêtres dans le texte italien, sont très nettement abrégés. Joulet ajoute, en revanche, une longue scène de déclaration amoureuse, qu'il insère entre le moment où Armide emporte Renaud sur son île et celui où Charles et Ubalde, les deux chevaliers partis à la recherche de ce dernier, surprennent leurs ébats. Cette scène, qui fait plus d'une cinquantaine de pages sur les quatre cents que compte le volume au total, vient combler un vide de l'épopée dans laquelle l'histoire d'amour d'Armide et Renaud n'est rapportée qu'indirectement (via un narrateur intradiégétique, pour le récit du coup de foudre, ou via le point de vue de Charles et Ubalde les observant à la dérobée) avant la rupture proprement dite. Elle est représentative de l'inflexion sentimentale que donne le romancier à cet épisode de $L a$ Jérusalem délivrée. Alors que chez Le Tasse l'amour d'Armide pour Renaud est concupiscent et asymétrique (puisqu'elle le séduit en l'ensorcelant), le romancier met en scène une affection réciproque et une héroïne chaste qui repousse à plusieurs reprises les propositions sexuelles de son amant.

5 Cette inflexion sentimentale se traduit également dans le traitement des discours. Suivant la mode du temps, Joulet adopte en effet un style pathétique flamboyant et très orné qui ne dépare pas du style Nervèze dont il est le contemporain. La voix narratoriale fait l'éloge, à plusieurs reprises, de l'éloquence passionnelle et envoûtante d'Armide présentée comme une oratrice hors pair, excellant dans le maniement des âmes :

Voyez un peu je vous prie combien la beauté, les larmes, \& l'eloquence d'une jeune Dame, ont de puissance sus nos Ames : ce sont des raisons ausquelles il n'y a point de responces: des demandes qui ne reçoyvent point de refus; des forces qui ne trouvent point de deffences : chasque parole qui sort de la bouche de la beauté est une chaisne qui attire, qui lie, \& qui serre les Ames avec des nœuds indissolubles: les pierres s'esmeuvent de ses larmes, les rochers sentent la douleur de ses peines, les vents les plus sourds escoutent ses plaintes, les elements luy obeïssent, la raison luy cede, les hommes l'adorent, les Anges la reverent, la terre \& le Ciel se sousmettent souz ses loix, \& soubz son obeïssance. 
Ceste belle Dame donc ayant envie de se faire aymer des plus grands de l'armée, pensa que la premiere chose qu'elle devoit faire, estoit de remarquer leurs humeurs, \& leurs inclinations, afin de pouvoir accommoder ses paroles, ses discours \& ses actions selon le goust de leurs opinions, sçachant bien qu'il n'y a point de prise si seure sur les volontez des hommes, que la cognoissance de leurs passions, comme fondements principaux sus lesquels on peut puis apres bastir toutes sortes de menées \& de negociations. Et pource que ceste cognoissance ne se peut acquerir à la première veue, elle se laissa librement accoster de tous ceux qui la voulurent entreprendre, \& mesme essaya d'en faire venir l'envie. [...]

Voyla comme la belle Armide, avec une façon si differente, alloit enyvrant les Ames, \& ensorcelant les volontez, tantost avec des ris \& des joyes, tantost avec des larmes $\&$ des souspirs ${ }^{10}$.

Si le premier de ces extraits est une amplification du texte italien ${ }^{11}$, le second est une addition de Joulet, qui prend la forme d'une véritable petite leçon de rhétorique sur la manière de sonder et disposer son auditoire pour obtenir son assentiment. Pour donner voix aux passions "muettes », le romancier ajoute également des discours au texte du Tasse, comme la longue séquence de dialogue amoureux que nous venons d'évoquer, et il amplifie un certain nombre de discours des passions. A contrario, les discours moins émouvants ou à visée didactique sont souvent rapportés au discours indirect, à l'instar des remontrances qu'adressent Ubalde puis l'ermite à Renaud ou des échanges entre Renaud et Godefroy. La scène de rupture entre Armide et Renaud, rappelé à sa mission guerrière par les deux chevaliers, illustre de manière exemplaire ce travail d'amplification qui touche aussi bien les discours du narrateur que ceux des personnages. Après l'évanouissement d'Armide, Joulet introduit ainsi un long psychorécit de Renaud qui le montre en train d'hésiter entre l'amour et la fuite :

Renault voyant la belle Armide estenduë sur la terre avec tous les signes apparents de la mort, demeura si estonné de la nouvauté de cest accident, qu'il ne sçavoit à quel party se resoudre car considerant que le desplaisir qu'elle ressentoit de son absence estoit la seule cause qui l'avoit reduite en ceste extremité, il voyoit bien que c'estoit une cruauté non encore veuë ny presqu'imaginée de la laisser en ceste façon esvanoüye sur le bord de la mer auparavant que sçavoir si elle estoit vive ou morte, \& quand le bien qu'elle luy vouloit (dont il voyoit d'aussi asseurez que pitoyables tesmoignages) ne l'auroit point particulierement obligé de l'assister en un inconvenient si digne de secours, la seule courtoisie qui nous rend redevable d'un si sainct office envers ceux mesmes que nous n'aurons jamais cogneuz, lui auroit bien forcé avec toutes sortes de douces \& charitables contraintes, \& pourtant qu'il ne pouvoit l'abandonner tant qu'elle eust recouvert \& l'esprit \& la vie, ou qu'il eust peu rendre à son corps les derniers honneurs que nous devons à nos amis qui se sont esloignez de ce monde : De demeurer aussi \& d'attendre le temps qu'il falloit pour l'esclaircir des doubtes qui tenoyent son ame suspenduë entre la crainte \& l'esperance de sa vie, il jugeoit bien que c'estoit chose qu'il pourroit fort malaisément obtenir de ces Gentils-hommes qui estoient avec luy lesquels n'estant touchez d'autre passion que du seul desir de le ramener en l'armée Françoise, n'attendoyent autre chose pour le faire embarquer que la fin de ceste amoureuse conference : Tellement que si le desir de la secourir \& les images de son affection, qui agitoyent sa memoire avec tant de doux \& chers mouvements, luy conseilloyent de demeurer avec ceste Pauvre Princesse (à la seule veuë des beautez de laquelle il sembloit que les rochers d'alentour s'animoyent \& s'eschauffoyent des feux d'amour) son honneur \& la dure necessité sourde \& aveugle née aux douces semonces de la pité le forçoit de s'en aller. De façon qu'apres qu'il eust quelque temps supporté le combat qui se faisoit en son entendement, entre l'envie qu'il avoit de demeurer aupres d'elle, \& la volonté de s'en esloigner, \& apres avoir mille $\&$ mille fois jetté les yeux de charité sur ceste pauvre Princesse, \& mille fois ceux de 
son honneur sur le vaisseau qui l'attendoit il se sentit en un coup emporter à la resolution de la laisser ${ }^{12}$.

Cette séquence développe, comme c'est fréquemment le cas dans le roman, une courte notation psychologique du texte italien, et précédée ici par une figure de suspension, qui court sur quatre vers chez Le Tasse et trois phrases chez Vigenère :

Or che farà? dée su l'ignuda arena

Costei lesciar così tra viva e morta?

Cortesia lo ritien, pietà l'affrena,

Dura necessità seco ne l' porta ${ }^{13}$.

Mais que fera-il ? Lairra il ainsi sur la greve ceste pauvrete à demy morte ? La

courtoisie le retient; la pitié l'empesche d'aller; mais la contrainte le ravist \&

emmeine avec soy ${ }^{14}$.

Peu après, un autre psycho-récit délibératif amplifie cette fois, non une notation psychologique, mais la plainte que prononce Armide au réveil de son évanouissement. P. Joulet allonge non seulement cette scène de trois autres plaintes, quand, une fois Renaud parti, Armide retourne dans son château sur les lieux où se sont déroulées ses amours, mais il augmente substantiellement les quatre discours principaux qui se succèdent dans cette scène, comme nous allons le voir maintenant en détails.

\section{De Virgile au Tasse : Armide, nouvelle Didon}

Afin d'apprécier l'adaptation proposée par Joulet, il convient d'abord de se pencher sur la plainte d'Armide chez le Tasse que nous analyserons ici dans la traduction de Vigenère. Cette traduction a en effet le double avantage de suivre de très près la leçon italienne ${ }^{15}$, excepté une petite amplification dans les imprécations finales, et d'être en prose (alors que la déploration de Tancrède est versifiée) ce qui facilitera la comparaison avec le texte de P. Joulet.

$7 \mathrm{Au}$ chant XVI de la Jérusalem délivrée, la scène entière de rupture entre Armide et Renaud est très visiblement imitée des adieux d'Énée et de Didon au livre IV de L'Énéide. Chez Le Tasse et Joulet, comme chez Virgile, le héros est rappelé à sa mission par l'entremise d'une puissance supérieure et il décide de partir sans en informer son amante qui, désespérée puis furieuse, le poursuit jusqu'au rivage pour tenter de le retenir sans succès. Dans ces trois textes, l'enchaînement des discours est le même à peu de chose près : après une prière (1. petitio) visant à le convaincre de rester, pour Didon, et de l'emmener avec lui, pour Armide, le héros refuse cette requête en tentant d'apaiser (2. conciliatio) son amante qui se met en colère et le congédie (3. exprobratio). Dans les trois cas, la requête liminaire de la protagoniste est déjà un discours de reproches qui a pour fin de renouer l'alliance avec son amant. Implorant Énée de rester, Didon formule le vœu d'avoir un enfant de lui tandis qu'Armide demande à Renaud qu'il l'emmène avec elle sur le champ de bataille et dit vouloir renoncer à sa foi. De la sorte, le passage de l'expostulatio («discours de reproches» à un ami) à l' exprobratio ( " condamnation sans appel » contre un ennemi ${ }^{16}$ ) traduit une augmentation de la colère de la protagoniste et de l'intensité émotionnelle de la scène. En revanche, la suite diffère sensiblement selon les trois auteurs. Tandis que Didon tourne les talons et demande ensuite à sa sœur, Anne, d'obtenir d'Énée un délai de quelques jours, Armide s'évanouit et, se réveillant après l'abandon de Renaud, prend la décision de se venger de lui. Joulet augmente cette scène de trois discours de lamentations d'Armide sur ses amours passées avec Renaud. Le tableau suivant donne un aperçu synthétique des similitudes et différences dans l'enchaînement des discours : 


\begin{tabular}{|c|c|c|c|}
\hline $\begin{array}{ll}\text { Genre } & \text { de } \\
\text { discours } & \end{array}$ & Virgile, Énéide, IV & $\begin{array}{l}\text { Le Tasse, La Jérusalem } \\
\text { délivrée, chant XVI }\end{array}$ & Joulet, Les Amours d'Armide \\
\hline $\begin{array}{l}\text { Expostulatio sous } \\
\text { la forme d'une } \\
\text { petitio }\end{array}$ & $\begin{array}{l}\text { Didon demande à Énée } \\
\text { de rester }\end{array}$ & $\begin{array}{l}\text { Armide demande à } \\
\text { Renaud de l'emmener } \\
\text { avec lui }\end{array}$ & $\begin{array}{l}\text { Armide demande à Renaud de } \\
\text { l'emmener avec lui }\end{array}$ \\
\hline Conciliatio & $\begin{array}{l}\text { Énée tente d'apaiser } \\
\text { Didon et lui demande } \\
\text { d'accepter son départ. }\end{array}$ & $\begin{array}{l}\text { Renaud } r \text { tente } \\
\text { d'apaiser Armide et lui } \\
\text { demande d'accepter } \\
\text { son départ. }\end{array}$ & $\begin{array}{l}\text { Renaud tente d'apaiser } \\
\text { Armide et lui demande } \\
\text { d'accepter son départ tout en } \\
\text { lui promettant de revenir. }\end{array}$ \\
\hline \multirow[t]{3}{*}{ Exprobratio } & Didon à Énée & Armide à Renaud & Armide à Renaud \\
\hline & $\begin{array}{l}\text { Petitio: Didon demande } \\
\text { à Anne d'intercéder en } \\
\text { sa faveur auprès d'Énée }\end{array}$ & $\begin{array}{l}\text { Plainte délibérative } \\
\text { d'Armide (seule) qui } \\
\text { décide de se venger }\end{array}$ & $\begin{array}{l}\text { Plainte délibérative d'Armide } \\
\text { (seule) qui décide de se venger }\end{array}$ \\
\hline & & & $\begin{array}{l}\text { Lamentations d'Armide } \\
\text { (seule) sur ses amours passées }\end{array}$ \\
\hline
\end{tabular}

8 La deuxième plainte d'Armide, sur laquelle nous allons nous pencher plus en détails à présent, est une réécriture très fidèle du discours de rupture de la reine de Carthage, comme l'indique d'ailleurs Vigenère dans ses annotations.

Virgile, exprobratio de Didon à Énée, vers Le Tasse, exprobratio d'Armide à Renaud, 365-387, traduction de Desfontaines, 1743

traduction de Vigenère, 1595 
[1] Perfide, lui dit-elle, transportée de colère, tu n'es ni le fils d'une Déesse, ni du sang de Dardanus. L'affreux Caucase t'a enfanté sur des rochers arides, et tu as sucé le lait des Tigresses d'Hyrcanie. Car qu'ai-je à dissimuler, et dois-je attendre que tu pousses l'offense plus loin? Le barbare a-t-il été touché de mes pleurs? A-t-il versé quelques larmes. A-t-il daigné regarder son amante, et être sensible à sa peine? Que te dirai-je enfin? La Déesse Junon qui me protège, et Jupiter même ont horreur de ta perfidie. [2] Hélas à qui se fier? Je l'ai recueilli dans mes États après son naufrage, et je l'ai associé à mon empire; j'ai sauvé ses vaisseaux échoués; j'ai arraché ses compagnons des bras de la mort. Enfin, je suis assez insensée pour l'aimer. [3] L'ingrat pour récompense prétexte maintenant des Oracles d'Apollon; et l'interprète des Dieux, qui on l'en croit, a traversé les airs, pour lui annoncer les redoutables ordres de Jupiter. Comme si les Dieux s'abaissaient à de pareils soins, et que les choses humaines fussent capables de troubler leur repos. [4] Je ne daigne pas te confondre, et je ne te retiens plus. Que les vents te conduisent dans ton Italie: va chercher à travers les ondes cette terre où tu dois régner. Si les Dieux justes ont quelque pouvoir, j'espère qu'un naufrage vengeur te fera échouer contre des rochers. Alors tu regretteras Didon, et tu l'appelleras vainement à ton secours: absente, je te poursuivrai la flamme à la main; et lorsque mon âme se sera envolée de mon corps glacé, mon ombre attachée à tes pas fera ton supplice, [cruel]. Dans le séjour des morts je serai informée de tes malheurs, et je m'en réjouirai ${ }^{17}$.
[1] Va ce ne fut oncques la belle Sophie qui t'enfanta, ny n'as point esté engendré du valeureux sang Attien: les tempestueuses \& impitoyables ondes des Syrthes t'ont produit tel que tu es \& les glacées crouppes du mont Caucase: les Hyrcaniennes Tygres furent les nourrisses qui t'allecterent. Que tarday je doncques? cest inhumain n'a point donné le moindre signe qu'il y eust une seule scintille d'humanité dans son cueur. A il seulment changé de teint, ny a mes tristes doleances surbaigné ses durs yeux d'une seule larme, ny boutté hors un seul souspir? [2.a] Quelle chose doibs je icy obmettre, ou quelle dire? Il s'offre estre mien, \& il s'enfuit de moy, \& me laisse. [2.b] o le brave, victorieux, qui veult si facilement oublier les offenses qu'on luy a faictes, \& pardonner de griefs forfaicts; mais oyez un peu comme il me conseille, oyez moy ce tant chaste Xenocrates, de quelle maniere il discourt d'amour. [2.c.] o ciel, ô Dieux pourquoy souffrez vous tant vivre de tels desloyaux detestables? \& au lieu que vous les devriez abismer, vous venez fouldroyer un pauvre clocher innocent, \& vos temples mesmes! [3] Va t'en doncq cruel, va t'en barbare, avec la paix que tu me laisses. Va t'en desormais en mall'heure, pervers trahistre inique affronteur perfide. Tu m'auras bien tost à tes tallons esprit despouillé de ce corps, vengeur de tes malheuretez; ombre qui te poursuyvra sans cesse. Tu m'auras invisiblement aux espaulles une nouvelle \& quatriesme furie pour te tourmenter en tous lieux, equippées de ses couleuvres remordantes, \& de ses brandons allumez. Je te feray aussi moleste \& ennuyeuse que je t'ay aymé. Et si ton destin porte que tu reschappes sain \& sauve des perils des ondes, \& qu'enfin tu parviennes à la bataille; là parmy les morts gisant plat estendu par terre, oultré de playes, \& prest à rendre les abbois, seras chastié de tes demerites. Tu auras beau appeler par son nom Armide, és derniers sanglots, je l'espere ainsi ${ }^{18}$.

9 Selon le découpage du rhéteur Ferrazzi (que nous suivons ici dans ses grandes lignes ${ }^{19}$ ), la plainte de Didon comporte quatre blocs principaux, qui correspondent en tous points au patron de l'exprobratio, tel que le définit Vossius ${ }^{20}$. Elle commence ex abrupto (c'est-àdire sans exorde, comme c'est souvent le cas dans les discours de colère) avec des reproches d'injustice et d'inhumanité adressés à Énée puis aux dieux. Suit l'énumération des bienfaits que Didon a accordés au héros troyen et qui ont pour fonction de souligner son ingratitude, puis la réfutation des causes du départ invoquées par Énée, dans son discours de conciliation. Enfin Didon congédie Énée et le maudit. La plainte d'Armide est constituée peu ou prou des mêmes arguments et a une structure 
très similaire. La première séquence est empruntée presque mot pour mot à celle du discours de Didon (seule manque la dernière phrase prenant les dieux à témoin). Dans la seconde séquence, l'énumération des services rendus par Didon à Énée est remplacée par celle des fautes de Renaud mais on y retrouve la réfutation du discours que vient de lui tenir le protagoniste. Armide reproche en effet à Renaud sa fuite ainsi que l'impudence et la mauvaise foi de sa réponse qui est soulignée ironiquement par l'antithèse « $O$ le brave, victorieux, qui veult si facilement oublier les offenses qu'on luy a faictes, \& pardonner de griefs forfaicts » et l'appellation antiphrastique «ce tant chaste Xenocrates». Ensuite, la prise à partie des Dieux, qui appuyait le premier argument de Didon, sert ici, nous semble-t-il, de transition vers la séquence suivante. En accusant les Dieux d'injustice Armide en appelle, en effet, à leur vengeance avant de maudire elle-même Renaud. Cette troisième et dernière séquence reprend dans le désordre les arguments de la péroraison de Didon. Le tableau ci-dessous présente schématiquement la structure séquentielle du discours du Tasse en comparaison avec celui de Virgile :

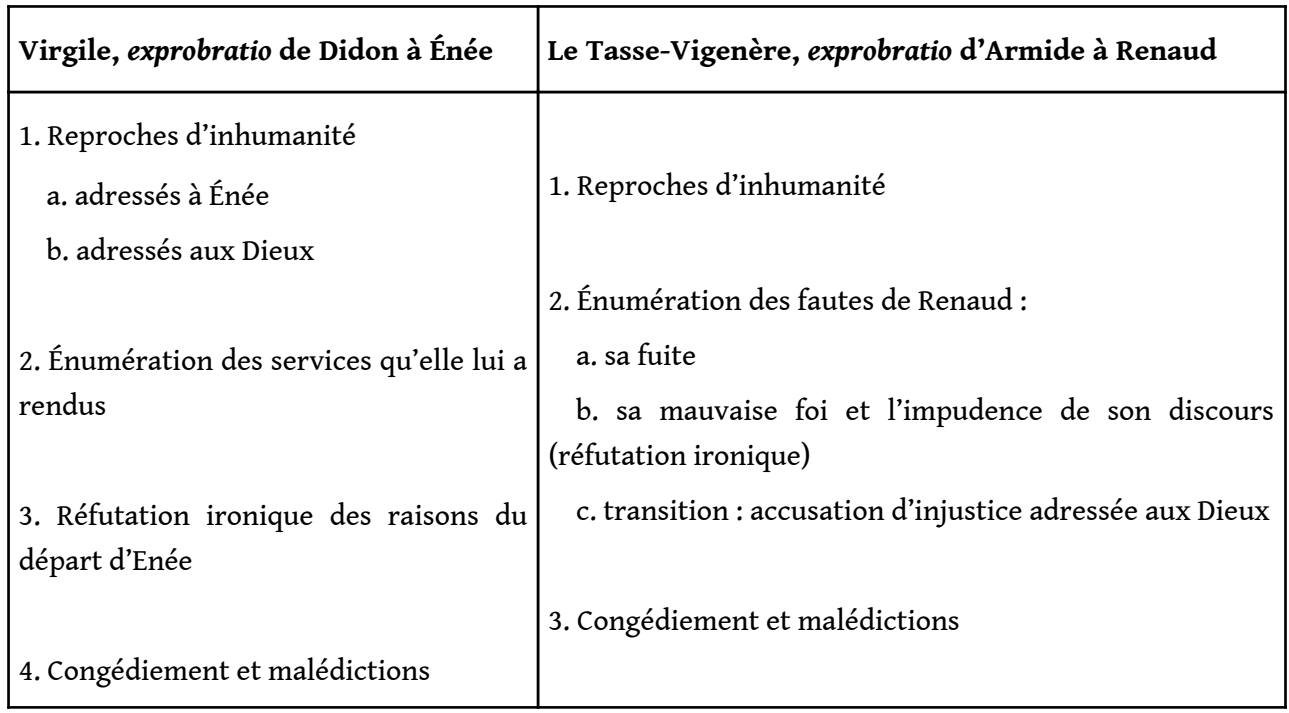

10 L'exprobratio d'Armide comprend donc une séquence en moins par rapport à celle de Didon. Cette suppression s'explique par le fait que, dans la plainte qui précède celle-ci, Armide avait déjà énuméré et exagéré les faveurs octroyées à Renaud en les présentant ironiquement comme autant de crimes. Conformément au genre de l'expostulatio, Armide incrimine ainsi Renaud indirectement en feignant de s'accuser elle-même, puis elle lui « offr[e] toutes espèces de bons offices ${ }^{21}$ » en proposant à Renaud de renoncer à sa foi, de le suivre comme esclave, de se couper les cheveux et de le protéger de son corps sur le champ de bataille.

\section{Du Tasse à Joulet : réécrire par amplification}

11 Dans Les Amours d'Armide, la plainte de colère de l'hérö̈ne est cinq fois plus longue que la version qu'en propose Vigenère. Tout en conservant la structure globale de l' exprobratio, Joulet amplifie en effet les séquences du texte original et en insère de surcroit, plusieurs nouvelles, de tailles et de fonctions diverses. Cette technique de réécriture par amplificatio rerum et verborum ${ }^{22}$ est bien rodée puisqu'il procède de même 
dans l'expostulatio d'Armide et dans la conciliatio de Renaud. Pour faciliter la lecture sans trop morceler le texte (dont la dynamique ne serait plus lisible), nous avons décomposé le discours de rupture en trois macro-séquences que nous analyserons successivement avant de proposer une interprétation plus générale des changements introduits par $P$. Joulet. Le début de chaque micro-séquence est signalé par un chiffre et une lettre entre crochets droits et les passages interpolés par Joulet sont encadrés des chevrons.

[1. Reproches adressés à Renaud et aux Dieux]

[1.a : reproches d'inhumanité] Meschant, vous n'estes point fils de la sage Sophie, il faut que le mont de Caucase qui est tousjours couvert de neiges, soit le Pere qui vous ait engendré en l'onde de la mer, lors que les vents l'ont renduë impitoyable, \& que vous ayez esté nourry du laict d'une Tygre ou d'une Lyonne, < puis que malgré toutes les prieres que je vous ay faictes, pour essayer de vous faire changer de dessein, ou de differer tant que je sois, où resolüe à recevoir les coups de vostre absence, ou préparée à souffrir ceux de ma mort, vous ayez bien eu le courage de me refuser > : comment vos yeux n'ont pas esté trempez d'une petite goutte d'eau froide, pour tant de larmes chaudes de rage que les miens ont respandues sur mon visage ? Vous estes demeuré ferme au millieu de toutes mes inquietudes, sourd à tant de justes plaintes, insensible entre tant de coups de mes douleurs : \& [1. b réfutation des déclarations de Renaud] < apres une si certaine cognoissance de la dureté de vostre courage, je dois croire que vous m'ayez jamais voulu du bien? Non, non, je manquerois de jugement en mesme temps que vous me manquez de foy, s'il m'en demeuroit quelque opinion : vostre infidélité a esté vostre seule affection, \& votre affection rien autre chose que vostre infidelité, où si ç'a esté de l'amour que ce que vous avez ressenty pour moy, ça esté un peu d'amour extrémement perfide \& trompeur, qui soubs des cendres froides \& glacée couvoit du venin dont mon ame (à mon dam trop credule) ne s'est peu garantir : que si je veux laisser dire à ma passion ce que ma juste douleur ou vos infidelitez lui conseille n'y a il quelque sorte d'oultrage que vous ne deviez attendre de moy. Et toutesfois nonobstant toutes ces preuves (dont je suis bien marrie de ne pouvoir plus douter) vous avez bien encore l'effronterie de me vouloir persuader que vous me voulez du bien. > [1.c accusation d'injustice adressée aux Dieux] o Dieux, qui estes icy presents, souffrirez vous plus que cest homicide de mon ame abuse plus long temps de vostre bonté \& de mon innocence? Vous estes offencez \& mesprisez comme moy, ne vengerez vous point bien tost ou vostre injure ou la mienne, ou l'une \& l'autre ensemble ${ }^{23}$ ?

12 La macro-séquence qui ouvre la plainte d'Armide est très nettement démarquée du texte du Tasse. On notera d'emblée qu'Armide vouvoie Renaud, selon les codes de la politesse mondaine, alors qu'elle le tutoyait dans le texte du Tasse. Dans le premier bloc [1.a], Joulet introduit simplement une amplification par les causes, qui relève de la catégorie plus générale de l'amplification par accumulation. L'ajout du segment : « puis que malgré toutes les prieres [...] vous ayez bien eu le courage de me refuser » vient souligner l'inhumanité de Renaud: il est inhumain car il est resté de marbre aux prières d'Armide. Le second mouvement [1.b] comporte une première différence avec le texte du Tasse puisqu'Armide ne reproche plus sa fuite à Renaud. De fait, chez Joulet, Renaud a promis à Armide de revenir dans un discours de conciliatio, qui est très nettement infléchi vers les codes de l'amour courtois. Ensuite, la réfutation des déclarations d'affection de Renaud est amplifiée par gradation: pour Armide, l'indifférence de Renaud montre qu'il ne lui a jamais voulu de bien et, qu'en réalité, il ne l'a jamais aimée. Ce constat est exagéré (au sens rhétorique du terme) par la figure du chiasme " vostre infidélité a esté vostre seule affection, \& votre affection rien autre chose que vostre infidelité ", puis à l'intérieur de la proposition concessive par la métaphore du concret pour l'abstrait : « soubs des cendres froides \& glacée couvoit du venin ». La troisième micro-séquence [1.c], dans laquelle Armide réclame vengeance 
aux Dieux, se signale à l'inverse par la concision et l'atténuation du propos. Tandis que, chez Le Tasse, Armide accusait le ciel d'injustice: "au lieu que vous les devriez abismer, vous venez fouldroyer un pauvre clocher innocent, \& vos temples mesmes!", ici, comme Didon, elle se contente de mettre au jour l'affront qui leur est fait. L'image de la foudre, présente dans le texte italien, disparaît pour réapparaître incidemment dans la séquence suivante où elle qualifie Renaud: "que vostre corps soit froissé de mille coups de foudre \& de tonnerre ». On relève cependant une petite amplification lexicale avec une périphrase dans le plus pur style Nervèze : "cest homicide de mon ame ». L'écart avec le texte du Tasse est à relier ici à l'atténuation de la dimension religieuse, qui caractérise le roman de P. Joulet, et qui apparaît à nouveau dans la double mention du Dieu Amour, dans la suite du discours. En effet, non seulement Armide ne renonce pas explicitement à sa foi dans l'expostulatio qui précède ${ }^{24}$ mais sa conversion, à la fin du roman, est simplement évoquée par le narrateur sans faire l'objet d'une scène à part entière, comme chez Le Tasse.

[2] Justification de sa colère]

$<$ [2.a] Mais helas que dy-je ? Est-il bien possible que je puisse jamais desirer la vengeance d'une personne que j'ay si esperduëment aymée : Quel tourment puis je souffrir assez cruel pour me faire tant soit peu blasphemer contre une seule de vos persecutions; Si j'ay eu plaisir d'estre des esleuz de vostre affection, j'auray la gloire d'estre des martyres de vostre haine, martyr dy-je à qui le seul respect ostera la voix au milieu de mes plus douloureux tourments, si ce n'est pour chanter des loüanges à vostre beau nom, lors que je seray au plus fort du supplice. Et quand mon esprit troublé des violences de mes maux, se seroit laissé emporter au desir de vengeance, la moindre estincelle de tant de sainstes flames qui sont en mon cœur seroit assez forte pour addoucir la plus vindicative de mes volontez: pardonnez moy donc s'il vous plaist mon cher (\& malgré vous) bien aymé Renault : Donnez à l'impetuosité de ma cholere l'inconsideration de mes injurieuses paroles : pensez que le coup de vostre absence a esté si rude qu'il ne m'a pas esté possible avec toutes les forces du respect que je vous doy, d'empescher à la douleur de les tirer de ma bouche : Pardonez s'il vous plaist à la pauvre Armide, ou (pour mieux dire) à tous ses desespoirs : aussi bien faut il que je confesse qu'en vous souhaittant $d u$ desplaisir, mes veuz sont si esloignez de mes paroles, que mon ame ne craint rien de plus au monde, sinon qu'il vous arrive le mal que je vous desire au chaud de ma cholere : je changeroy donc maintenant, non de volonté, mais de la façon de me plaindre, [2.b] je suis contente flattant ma douleur (avec des vaines esperances, mais de trop certains desplaisirs) de croire que vostre affection n'est point encore du tout esteinte \& amortie : mais aydez un peu à ma faulse creance : car si je l'ay prise, helas ! ce n'est que de mes desirs, tous seuls, \& non de pas une de vos actions. Si donc vous avez encore un peu de bonne volonté, voire presque de souvenance d'en avoir jamais eu pour moy, pouvez vous bien contre les persuasions \& les prieres que je vous ay faictes depuis que je suis aupres de vous, \& si hors de moymesme, me refuser ce que je vous demande ? Demeurez, demeurez donc avec moy, changez de dessein, ou le differez pour quelques jours : [2.c] mais pourquoy veux je encore malgré une si veritable cognoissance de vostre mauvais naturel, me persuader que vous vouliez jamais plus rien faire pour moy: Mon ame sera elle tousjours pipée de ses propres passions ? O miserable Armide, à quelle extremité suis je reduite, puis que mes desirs \& mes volontez me trahissent, \& me celent mes peines, \& mes pertes, \& que je n'ay plus rien qui m'en parle à la verité que le seul ressentiment de la douleur qu'elles m'apportent. Ne me trompez donc plus s'il vous plaist dictes-moy librement que vous me voulez du mal, \& puis que les feux de vostre cœur se sont convertis en glaces de hayne, ne vous mettez plus en peine de les couvrir avec des flammes feintes \& supposées: contentez vous de m'avoir trompée jusques icy soyez si vous voulez encores plus perfide, mais pour le moins ne soiez plus si dissimulé : faictes que vos paroles s'accordent mieux avec vos 
pensées : aussi bien ne cognoy je que trop le refroidissement de vostre volonté, les glaces y sont trop froides \& trop espesses : \& je seray encore si sotte de me laisser piper à vos paroles, les instruments malicieux des infidelitez \& dissimulations de vostre ame : \& je defendray les outrages à ma bouche. Non, non qu'elle die ce quil luy plaira, ma douleur est trop grande pour pouvoir plus estre ou muette ou discrette : \& puis que j'ay perdu m'asseure-je bien qu'elle ne me lairra point de regret de n'avoir eu mourant osé vous reprocher vos infidelitez, \& d'invoquer le Ciel avec la voix pitoyable de mes pensées innocentes, à ce que vostre corps soit froissé de mille coups de foudre \& de tonnerre, pour en jetter puis apres les membres funestes au vent de vos legeretez. Helas! me souvenant de la promesse que vous me faisiez toustes à ceste heure, de me venir retrouver, \& du conseil que peu apres vous me donniez d'assoupir mes flammes, \& d'en enterrer les cendres au mesmes lieu où elles ont esté si peu judicieusement ce dites vous allumées, que doy je dire de vous? ay-je pas une juste occasion bannissant de mon ame cest opiniatre respect, \& ceste forte reverence que je vous ay gardée, de vous dire que vous estes le plus trompeur, le plus meschant, le plus dissimulé, perfide de la terre, \& de prier le Dieu de l'amour que vous avez offensé, qu'au premier pas que vous ferez pour vous esloigner de moy, la terre s'ouvre \& vous engloutisse, \& que les vents avec lesquels vostre ame à une telle sympathie, vous eslevans en l'air sur leurs aisles, vous precipitent puis apres dans les plus creuses abysmes de la mer, pour supplice trop doux encore pour les tourments si apres que vous me faictes ressentir? vous me conseillez donc que je demeure icy toute seule, que je tue une affection si saincte \& si sacrée que la mienne, que j'amortisse des desirs dont les impatiences me sont si douces \& aggreables, que je retire ma volonté d'un lieu où le destin l'a portée avec tant de cheres \& precieuses violences. O remede plus cruel \& plus rigoureux que ne sont les blesseures de mon cœur, \& ses plus perilleuses maladies ${ }^{25}$ !>

13 Si jusque-là, le texte de Pierre Joulet suivait, à peu de choses près, la même disposition que le texte italien, la deuxième macro-séquence, qui représente la moitié du discours d'Armide, introduit un développement entièrement inédit qui s'intercale entre la séquence 2 et 3 du discours dans la version du Tasse. L'interjection liminaire "Helas » signale un revirement de la part d'Armide. L'exprobratio, plainte de colère, semble alors se muer en lamentation, plainte de douleur, comme l'indique d'ailleurs cette déclaration de la protagoniste : «je changeroy donc maintenant, non de volonté, mais de la façon de me plaindre ». L'expression du désespoir d'Armide ne dure pas, cependant, et l'indignation lui succède à nouveau au gré de multiples revirements : au désespoir succède l'espoir d'être toujours aimée puis à cet espoir le rappel de l'infidélité de Renaud, à la décision de faire taire sa douleur fait suite la reprise des récriminations. Ce jeu de contrepoints exhaussé par l'antithèse: «je suis contente flattant ma douleur (avec des vaines esperances, mais de trop certains desplaisirs) », l'interrogation: «mais pourquoy veux je encore malgré une si veritable cognoissance de vostre mauvais naturel, me persuader que vous vouliez jamais plus rien faire pour moy?", ou la correction : « je defendray les outrages à ma bouche. Non, non qu'elle die ce qu'il luy plaira ", relève de la dubitatio ou doute, figure pathétique par excellence, qui délimite ici à elle seule un bloc à part entière du discours. Imitant les volte-face d'une âme en proie à des passions contraires, ces revirements n'en suivent pas moins une argumentation bien articulée. Cette macro-séquence est en effet décomposable en trois sous-séquences qui forment autant d'arguments. Dans la première [2.a], Armide se dédit de son appel à la vengeance et déclare renoncer à sa colère. Dans la seconde [2.b], la protagoniste demande à Renaud de rester avec elle ou, à l'instar de Didon quand elle demande à Anne d'intercéder pour elle auprès d'Énée ${ }^{26}$, d'ajourner son départ. Dans la troisième [2.c], se rendant compte qu'elle se berce d'illusions, elle réitère ses 
récriminations (en partie ironiques) contre les mensonges de Renaud, développement qui est construit par amplification lexicale de la réfutation de la première séquence [1.b]. Ces trois mouvements, s'ils paraissent digressifs à première vue, servent tous à appuyer la proposition de l'exprobratio en justifiant la colère d'Armide qui emploie d'ailleurs le champ lexical de la justice : «ay-je pas une juste occasion bannissant de mon ame cest opiniatre respect, \& ceste forte reverence que je vous ay gardée, de vous dire que vous estes le plus trompeur [...] ». Sa colère est légitime car elle procède de son désespoir [1.a], car Renaud ne lui donne aucune assurance de son amour [1.b], car Renaud la trompe par ses belles paroles [1.c]. Dans ce dernier segment, on relève la métaphore filée des désordres météorologiques et du vent : « pour en jetter puis apres les membres funestes au vent de vos legeretez » et «qu'au premier pas que vous ferez pour vous esloigner de moy, la terre s'ouvre \& vous engloutisse, \& que les vents avec lesquels vostre ame à une telle sympathie, vous eslevans en l'air sur leurs aisles, vous precipitent puis apres dans les plus creuses abysmes de la mer ", qui reprend l'image de la tempête des passions très longuement développée, chez Joulet, dans l'expostulatio d'Armide ${ }^{27}$. Cette image, inspirée du texte virgilien, dans lequel l'union de Didon et Énée s'accompagne d'un orage figurant allégoriquement le déchaînement des passions, semble transposer sur le plan de l'élocution la situation narrative de l'Énéide.

\section{[3. Congédiement et malédictions]}

$<$ [3.a] Allez allez meschant, gardez ceste cruele misericorde, \& ceste malicieuse bonté pour une autre que pour moy, allez, si je suis si mal heureuse de ne pouvoir pas trouver le moyen de vivre avec vous, au moins ne seray je pas si miserable de ne pouvoir trouver celui qui me pourra plus doucement conduire à la mort: mais pensez vous avec ces malheureux conseils estre quitte de toutes vos perfidies à la justice vengeresse de l'amour, la divinité duquel vous avez si temerairement \& si malicieusement offensée se represente-elle point devant vos yeux? des tourments preparez aux ames ingrates, ne vous apportent-ils point de crainte \& d'apprehension? Quoy Renault, vous voulez vous separer de la pauvre Armide \& jettant au vent voz serments \& voz promesses, me laisser en ce lieu desert sans vous, voire sans moy-mesme? Ha que voy-je Renault, vous vous en allez? je ne puis plus vous arrester d'avantage, vous me voulez laisser auparavant que j'aye peu mourant de regret, m'empescher une veuë si douloureuse:> [3.b] Allez, allez perfide, allez où vous voudrez, \& emportez avec vous le mesme repos que celuy que vous me laissez maintenant, < que ce mesme desespoir qui m'enflamme de rage, vous accompaigne en vostre voyage, que la cholere boüille tousjours dedans vos veines : que mille regrets comme vers affamez rongent incessamment vostre cœur, sans qu'ils se rassasient jamais, qu'un monde de soucis veillent continuellement à l'entour de vostre ame : que vos yeux ne voyent que fantosmes espouvantables : que vostre imagination soit sans cesse agitée de l'horreur des peines que vous meritez, que vostre entendement ne prevoye que malheur pour le reste de vos jours : que vostre volonté ne soit plus enflammée que de feux, de cholere \& de rage contre vous mesmes, au lieu de tant de cheres flammes qui l'ont pour quelque temps si doucement eschauffée. > Allez \& vous asseurez que vous n'irez guere loing d'icy que vous ne me voyez bien tost aupres de vous, comme ombre effroyable de la mort, ou comme un Demon plein de terreur \& de frayeur, qui vous suyvra par tout, non pour vous demander encore vostre affection, que vostre infidelité commence à me rendre si odieuse, mais bien pour agiter \& troubler vostre esprit, avec mille furies \& mille flambeaux ardents de haine: que si quelque destin favorisant une vie si pernicieuse que la vostre vous fait eschapper des perils de la mer que vous allez traverser, \& qu'en despit des orages, des vents \& des tempestes que le Ciel tient prests pour venger le tort que vous me faictes vous arriviez en vostre armée, au moins esperay je bien qu'à la premiere escarmouche où vous irez vous n'eviterez point la punition que merite vostre infidelité \& vostre ingratitude, \& que je vous 
feray bien payer par la rigueur des supplices \& des coups mortels que vous y recevrez, la peine qui vous est deuë, \& qu'estant blessé \& tombé par terre entre les morts \& le sang des meschans de vos troupes, en rendant les derniers abois de la vie, vous vous souviendrez d'Armide, \& que vous appellerez \& invoquerez son nom avec autant de desir d'en recevoir des remedes pour vos playes, que vous me laissez aujourd'huy de regret de m'en refuser pour les miennes : \& lors à vostre exemple je seray sourde à vos cris impitoyables au milieu de vos plus cuisantes douleurs. < [3.c] Mais las! d'où est-ce que je puis prendre ces vaines \& piteuses esperances avec lesquelles j'entretiens, ou pour mieux dire, je trompe mon ame irritée ? auray-je bien encore assez de vie pour attendre ce jour, jour dy-je qui sera aussi favorable à mes haynes que celuy cy est funeste à mon amour? Non non, je n'auray jamais assez de force pour supporter le faiz de tant de lourds ennuis que ceux de ceste cruelle separation. Il vaut doncques mieux quittant le desir de la vengeance avec laquelle je me vays trompant, me resoudre à me donner la mort auparavant que je la reçoive de vostre absence, ou de vostre hayne : \& puis que le Ciel me veut oster le moyen de pouvoir jamais plus vivre avec vous, avec vous dy je, où j'avois ce me sembloit si heureusement logé tous mes desirs \& mes passions que je perde quand \& quand ceste inutile, opiniastre \& desja importune volonté de vivre jamais plus avec moy mesme ${ }^{28}$. >

Nous avons choisi de faire débuter la troisième macro-séquence à la première occurrence du verbe aller à l'impératif, qui marque communément le congé donné à la personne ennemie dans une exprobratio, et qui est répétée six fois par Armide. Le premier mouvement [3.a] de ce bloc sert de transition entre les deux macro-séquences. Après avoir congédié son amant, Armide revient à nouveau sur la trahison de Renaud et ses paroles mensongères avant de se rétracter à nouveau et d'essayer de le retenir : "Quoy Renault, vous voulez vous separer de la pauvre Armide [...] ? ». La péroraison commence véritablement dans le deuxième segment de cette séquence avec les imprécations d'Armide imitées du Tasse. Dès le début de ce second mouvement [3.b], le terme antiphrastique de « repos » est amplifié par accumulation des circonstances de la personne qui décrivent l'enfer psychique qu'Armide souhaite à Renaud et que ponctue une série d'homéotéleutes: «que ce mesme desespoir qui m'enflamme de rage vous accompaigne en vostre voyage, que la cholere boüille tousjours dedans vos veines, que mille regrets comme vers affamez rongent incessamment vostre cœur, sans qu'ils se rassasient jamais ». À ces imprécations, qui reprennent ensuite tous les motifs du texte italien (la poursuite de la furie, le naufrage, le champ de bataille, l'invocation du nom d'Armide par Renaud mourant) en augmentant le matériel verbal, succède un troisième mouvement [3.c], ajouté par Pierre Joulet. Dans un ultime revirement, Armide, désespérant de se voir jamais vengée, en appelle à la mort pour mettre fin ses souffrances, à la malédiction succède le souhait. On peut lire dans ce dernier segment, la continuation de la figure de la dubitatio et une amplification par les conséquences du précédent argument: ne pouvant ni se venger, ni supporter la séparation, Armide préfère encore mourir. Si cet appel à la mort, préparé dès la deuxième séquence du discours (« au moins m'asseure-je bien qu'elle ne me lairra point de regret de n'avoir eu mourant osé vous reprocher vos infidelitez»), ne figurait pas chez Le Tasse, il surimprime à la plainte d'Armide le final de Didon qui imagine sa vengeance post mortem: «Dans le séjour des morts, je serai informée de tes malheurs, et je m'en réjouirais ». 


\section{De la condamnation sans appel à la lamentation}

15 L'analyse montre que, s'il ne pouvait ignorer la traduction de Vigenère paru chez le même éditeur, Joulet ne travaille pas à partir de celle-ci : soit qu'il n'en ait pas eu connaissance au moment de la rédaction de son roman ou qu'il écrive directement à partir du texte italien. On remarque aussi que le romancier transpose la scène de rupture en ayant visiblement en mémoire celle de Didon et Énée, qui se surimprime au discours d'Armide par le truchement de plusieurs éléments (l'appel à la vengeance des Dieux, l'ajournement du départ de Renaud, l'image de la tempête, l'appel à la mort).

En regard du texte du Tasse et de la traduction de Vigenère, la réécriture de $\mathrm{P}$. Joulet se distingue par la recherche d'expressivité et l'intensification du pathos. L'ajout de la figure de la dubitation qui n'apparaissait qu'ultérieurement chez Virgile et chez le Tasse (dans le soliloque de Didon lors du départ d'Énée ${ }^{29}$ et dans la troisième plainte d'Armide chez le second) et la réitération des mensonges de Renaud, par amplificatio verborum et rerum, peignent les revirements et l'obsession d'une conscience désemparée. Passant de l'expression de la colère à celle du désespoir, de l'excitation de l'indignation à celle de la pitié, P. Joulet lâche la bride au pathos au moyen de la technique de l'amplification, qui vise précisément à émouvoir ${ }^{30}$, et d'une ornementation voyante. Les passages interpolés par P. Joulet sont particulièrement imagés et font résonner les figures de mots et même les figures "gorgianiques » antithèses et homéotéleutes - traditionnellement proscrites des mouvements véhéments ${ }^{31}$.

17 La coloration doloriste, que le romancier confère à cette plainte en la concluant par un appel à la mort, va de pair avec l'inflexion sentimentale du roman. Elle traduit un attendrissement $d u$ caractère des protagonistes qui contribue à la revalorisation du personnage d'Armide. C'est Médée qui se double d'Ariane, en laissant percer, derrière la furie maléfique, les accents douloureux de la femme abandonnée. Sur un plan rhétorique, l'atténuation de la colère d'Armide, notamment par la figure de la dubitation qui interroge la légitimité de l'expression de cette passion, correspond aussi, nous semble-t-il, à une question de convenance. Il est en effet malséant pour une jeune femme, et a fortiori pour l'héroïne d'un roman sentimental, de laisser libre cours à son ressentiment. De fait, si Jean Corbin donne encore voix à la fureur de la femme outragée, les romanciers qui adaptent cet épisode par la suite, comme Suzanne Nervèze dans La Nouvelle Armide (1645) et Antoine Gombaud Méré dans Les Aventures de Renaud et $d^{\prime} A^{\prime}$ inde $^{32}$ (1678), transformeront la condamnation sans appel en lamentation.

Les Amours d'Armide témoigne de la lecture romancée et néo-asianiste ${ }^{33}$ que l'on fait alors de La Jérusalem délivrée à l'orée du XVII ${ }^{\mathrm{e}}$ siècle et, plus largement, de la littérature italienne. Sous la plume de Pierre Joulet, l'histoire d'Armide et de Renaud devient une allégorie des séductions de l'éloquence. Déjà, le texte italien opposait une oratrice hors pair à un jeune homme qui, après avoir succombé à ses charmes, doit faire la preuve, par deux fois, qu'il est capable de résister aux chants des « sirènes ${ }^{34}$ » (ceux d'Armide et des créatures de son royaume puis ceux des figures enchantées de la forêt) pour regagner sa place dans le camp chrétien. En germe chez Le Tasse, cette lecture métadiscursive est actualisée dans le roman de Joulet par le déploiement d'une rhétorique pathétique particulièrement ornée et la mise en valeur des dons oratoires d'Armide, qui paraissent plus dangereux que sa beauté physique et ses enchantements. 
Princesse orientale, l'artificieuse Armide y apparaît moins comme une figure diabolique que comme l'incarnation d'une rhétorique néo-asianiste à laquelle, malgré sa défaite finale, le roman offre ici une tribune de choix.

Illustration flamboyante de la vogue du "pathos frénétique ${ }^{35}$ » importé d'Italie, l' exprobratio d'Armide est aussi l'un des derniers exemples romanesques de plainte de colère imitée de celle de Didon ${ }^{36}$. Si l'intertexte virgilien est encore très prégnant dans le roman du $\mathrm{XVII}^{\mathrm{e}}$ siècle $^{37}$, on privilégie des passions moins violentes et on n'abandonne plus guère d'amantes éplorées. Les discours de reproches ont désormais plutôt pour motifs la trahison ou l'infidélité - supposées - de la personne aimée. Quand, dans L'Académie de l'art poétique (1610), P. Deimier propose une « imitation libre » de l'histoire de Didon et Énée pour la transformer en récit sentimental, il substitue aux imprécations de la reine de Carthage une lettre de jalousie de l'époux à sa femme qu'il croit adultère ${ }^{38}$. Ainsi, dans Les Amours infidelles (1625) de Claireville ${ }^{39}$, où la première histoire s'intitule "Amours de Didon \& d'Enée ", la fureur de l'héroïne virgilienne est très atténuée et même dégradée par une intrigue galante dans laquelle la protagoniste soupçonne Énée d'une relation amoureuse avec sa sœur, Anne. Cette inflexion de la plainte d'indignation procède à la fois de la célébration d'un couple de protagonistes ainsi que de l'hérö̈sation des personnages féminins. Par ailleurs, sur le plan des modèles d'éloquence pathétique, il nous semble caractéristique que la référence au texte antique s'opère indirectement dans Les Amours d'Armide, par l'intermédiaire d'une œuvre en langue vulgaire ${ }^{40}$. De même, dans Les Amours d'olympe et de Birène (1599), A. de Nervèze réécrit la plainte d'Ariane par le truchement d'un épisode du Roland Furieux, dans lequel L'Arioste transposait très fidèlement l'héroïde ovidienne. Ces exemples, comme celui de L'Astrée qui s'ouvre sur une exprobratio de l'héroïne à Céladon imitée de la lettre d'Oriane au chevalier Amadis, montrent qu'une culture rhétorique proprement romanesque, et distincte des modèles d'éloquence scolaires, est alors en voie de constitution.

\section{NOTES}

1. La première en prose de Blaise de Vigenère (Paris, L'Angelier, 1595), la seconde en vers de Jean du Vigneau (Paris, Guillemot et Gilles, 1595) et la troisième de Pierre de Brach (Paris, L'Angelier, 1596) qui traduit seulement les chants II (histoire d'olinde et Sophronie), IV (Armide dans le camp des chevaliers chrétiens), XII (la mort de Clorinde) et XVI (histoire de Renaud et Armide).

2. Clorinde ou l'amante tuée par son amant (Paris, C. de Monstr'œil, 1597) d'un auteur anonyme ; Hierusalem assiégée, Où est descrite la delivrance de Sophronie, \& d'Olinde: ensemble les Amours d'Hermine, de Clorinde, \& de Tancrede (Paris, A. du Breuil, 1599) d'Antoine de Nervèze ; et Jerusalem regnante, contenant, la suite \& la fin des Amours d'Armide et d'Hermine (Paris, L'Angelier, 1600) de Jacques Corbin. Sur les réécritures françaises de La Jérusalem délivrée, voir la conclusion de R. Gorris-Camos dans L'Arioste et le Tasse en France au XVI siècle, Cahiers V. L. Saulnier 20, Éditions Rue d'Ulm, Presses de l'École normale supérieure, 2003, p. 257-274.

3. Pierre Joulet a également écrit deux textes moraux: Les Amours spirituels de Psyché (Paris, L'Angelier, 1606) et La Défense de l'inconstance (Paris, L'Angelier, 1608). 
4. N. Boileau, Art poétique, satire IX.

5. F. Panigarola, Il Predicatore, Venetia, Giunti, 1609, p. 240-241. À ce sujet, voir C. Mouchel, Cicéron et Sénèque dans la rhétorique de la Renaissance, Marburg, Hitzeroth, 1990, p. 92-111.

6. D. Bouhours, La Manière de bien penser dans les ouvrages d'esprit. Dialogues, Paris, Vve Sebastien Mabre-Cramoisy, 1687, p. 236.

7. P. Joulet, Les Amours d'Armide, Paris, L'Angelier, 1606, n. p.

8. B. de Vigenère, La Hierusalem du Sr Torquato Tasso, Paris, Abel L'Angelier, 1595, f. 305 vº.

9. N. Denisot procède de même dans L'Amant ressuscité de la mort d'amour (1555) où il réécrit le livre IV de L'Énéide. Voir F. Greiner, « De l'épopée à l'histoire tragique : la réécriture de L'Énéide dans L'Amant ressuscité de Denisot", L'Épopée et ses modèles de la Renaissance aux Lumières, F. Greiner et J.-C. Ternaux dir., Paris, H. Champion, 2002, p. 73-89 et l'article de J. Lecointe, « Les cadres rhétoriques de l'innutrition virgilienne dans le "roman sentimental": Hélisenne de Crenne et Théodose Valentinian » dans le présent numéro.

10. P. Joulet, op. cit., p. 57 et p. 61-71.

11. Le Tasse, Gerusalemme Liberata. Jérusalem délivrée, Édition bilingue, trad. G. Genot, Paris, Les Belles Lettres, 2008, vol. 1, IV, 83, p. 89: "Or che non può di bella donna il pianto, / ed in lingua amorosa $i$ dolci detti? / Esce da vaghe labra aurea catena / che l'alme a suo voler prende ed affrena." "Que ne peuvent les pleurs de belle dame, / et de bouche amoureuse les douces paroles? / Car une chaîne d'or sort des lèvres gracieuses, / qui à son gré captive et tient au frein les âmes. »

12. P. Joulet, op. cit., p. 319-322.

13. Le Tasse, op. cit., vol. 2, XVI, 62, p. 142 : «Que fera-t-il ? Faut-il que sur l'arène nue / il l'abandonne aussi morte que vive ? / Courtoisie le retient, pitié l'arrête, / dure nécessité avec elle l'entraîne. "

14. B. de Vigenère, op. cit., f. $224 \mathrm{v}^{\circ}$.

15. Voir F. Graziani, «Sur le chemin du Tasse : la fidélité du traducteur selon Vigenère, Baudoin et Vion d'Alibray ", L'Arioste et le Tasse en France au XVI ${ }^{e}$ siècle, Cahiers V. L. Saulnier 20, Paris, Éditions Rue d'Ulm, 2003, p. 203-216.

16. Fr. Goyet propose de traduire le terme d'expostulatio par «discours de reproches» et d' exprobratio par l'expression "condamnation sans appel ». Étudiant la définition et le patron de ces deux genres de discours dans les Institutiones (1605) et la Rhetorice contracta (1621) de Vossius, il explique que l'expostulatio est adressée à une personne amie tandis que l'exprobratio est adressée à un ennemi. Voir G. J. Vossius, Institutiones (1605), III, 17, « Le discours de reproches ", et 18, « Le discours de condamnation sans appel », éd. Fr. Goyet et trad. L. Vianès, Exercices de rhétorique 2 | 2013, URL : http://rhetorique.revues.org/187; et G. J. Vossius, Rhetorice contracta (1621), II, 27, «Les discours de reproches et de condamnation sans appel ", éd. Fr. Goyet et trad. L. Vianès, Exercices de rhétorique 2 | 2013, URL : http://rhetorique.revues.org/190. Voir aussi Fr. Goyet, «La rhétorique de Didon dans l'acte II : l'exprobratio au sein des vers 851-952 », Lectures de Jodelle. Didon se sacrifiant, éd. E. Buron et O. Halévy, Presses Universitaires de Rennes, 2013, p. 65-96.

17. Virgile, L'Énéide, trad. Desfontaines (Pierre François Guyot), Paris, Quillau, 1743.

18. B. de Vigenère, op. cit., f. $223 \mathrm{v}^{\circ}-224 \mathrm{r}^{\circ}$.

19. Voir le discours 31 des Exercitationes rhetoricae in praecipuas $P$. Virgilii Maronis orationes, quae in Aeneidum libris leguntur (1694) de Marco Antonio Ferrazzi, édition de Christine Noille en collaboration avec RARE (Rhétorique de l'Antiquité à la Révolution), en ligne à l'adresse : http:// rare.u-grenoble3.fr/spip/spip.php?article110.

20. G. J. Vossius, Institutiones (1605), III, 18, « Le discours de condamnation sans appel », op. cit. ; et G. J. Vossius, Rhetorice contracta (1621), II, 27, « Les discours de reproches et de condamnation sans appel », op. cit.; la plainte de Didon est l'un des exemples d'exprobratio le plus souvent cité par les rhétoriciens classiques, avec la plainte d'Ariane à Thésée de Catulle et l'héroïde de Médée à Jason d'Ovide. 
21. Selon l'expression de Vossius pour définir l'expostulatio dans les Institutiones, III, 17, op.cit., p. 2.

22. Sur la distinction entre ces deux types d'amplification et une description complète de leur fonctionnement, voir S. Macé «L'amplification, ou l'âme de la rhétorique. Présentation générale ", Exercices de rhétorique, 4 | 2014, URL : http:// rhetorique.revues.org/364.

23. P. Joulet, op. cit., p. 303-306.

24. Ce renoncement, qui était explicite chez Le Tasse, prend la forme d'une métaphore galante chez Joulet : "Allez vous en, quittez ces lieux ou vostre nom est si superstitieusement honoré, allez ruiner les fondements de ma foy \& de ma religion (si toutefois je puis plus avoir d'autre foy $\&$ d'autre religion que celle que m'ont donnée vos beaux yeux, bien cheres mais trompeuses idoles)» (op. cit., p. 280). P. Joulet propose ici un moyen terme entre la version originale et celle de Vigenère, qui gomme toute référence à la religion d'Armide dans ce discours et qui emploie le terme «foi » au sens figuré de «foi amoureuse »: "Va t'en donques, repasse la mer, combats, travaille ; romps ceste foy, que nous-nous estions reciproquement donné l'un à l'autre ; C'est moy mesme qui t'en recherche \& sollicite : mais que dis-je nostre ? ah non plus tienne, car il n'y a que moy qui la garde » (Vigenère, op. cit., f. $222 \mathrm{r}^{\circ}$ ).

25. Ibid., p. 306-312.

26. Énéide, IV, vers 416-437.

27. «On dit que les orages n'arrivent jamais sans quelque presage auparavant: mais ceste nouvelle tempeste de voz courroux m'ayant surprise sans que j'en aye peu descouvrir aucun signe en vos actions, me faict bien cognoistre qu'il y a quelque cruel destin, qui me vient voler tout mon bien, \& en menant avec luy un Aquilon de mal-heurs \& tous les vents aspres \& froids, me vient esteindre voz flammes en les allumant ... » (P. Joulet, op. cit., p. 283-284.)

28. Ibid., p. 312-317.

29. Énéide, IV, v. 595 : «Quid loquor ? aut ubi sum?»

30. Selon la définition qu'en donne Cicéron dans les Partitiones oratoriae, 27 (trad. H. Bornecque, Paris, Les Belles Lettres, 2002 [1924]) : «Quant à l'amplification, bien qu'elle ait sa place propre, souvent au commencement, presque toujours à la fin, on doit l'employer aussi dans tout le corps du discours, surtout à l'appui des preuves et des réfutations. Voilà pourquoi elle aussi est un puissant moyen de convaincre; en effet l'amplification est comme une argumentation passionnée ; l'argumentation instruit, l'amplification émeut. "

31. On attribue généralement cette proscription à Démétrios mais elle se retrouve également dans les traités des $\mathrm{XVI}^{\mathrm{e}}$ et XVII ${ }^{\mathrm{e}}$ siècles, comme par exemple dans le De arte rhetorica de C. Soarez : « quand il faut lutter par la violence, l'envie, la pitié, qui supportera celui qui s'emporte, celui qui pleure, et celui qui supplie avec des antithèses, des homéotéleutes et des figures similaires ?» (Séville, A. Pescioni, 1569, f. $54 \mathrm{v}^{\circ}$, notre traduction). Sur Démétrios, voir l'introduction de P. Chiron à son édition du Style, Paris, Les Belles Lettres, 1993.

32. S. de Nervèze, La Nouvelle Armide, Paris, Jean Pasle, 1645, p. 62-64 ; Antoine Gombaud Méré, Les Aventures de Renaud et d'Armide, Paris, Claude Barbin, 1678, p. 148-149. Dans ces deux textes, la plainte d'Armide est, par ailleurs, très abrégée.

33. Selon les analyses de J. Balsamo dans La Rencontre des Muses. Italianisme et anti-italianisme dans les lettres françaises de la fin du XVI ${ }^{e}$ siècle, Genève, Slatkine, 1992, p. 290-300.

34. Selon le terme qu'emploie Ubalde pour l'enjoindre à répondre à Armide qui le supplie de l'écouter avant de s'en aller : "Qual piú forte di te, se le sirene / vedendo ed ascoltando a vincer t'usi ? » «Or qui sera plus fort que toi, si les sirènes / voyant et écoutant, à vaincre tu t'éduques?» (Le Tasse, éd. cit., vol. 2, XVI, 41, p. 137).

35. L'expression est de Jean Lecointe dans «Le miel de la colère: la douceur des passions violentes à la Renaissance » (V. Duché-Gavet et al. éd., Du sucre, Biarritz, Atlantica, 2007, p. 69-87 et ici p. 81). 
36. On citera, comme exception notoire, les plaintes de Calypso dans Les Aventures de Télémaque (1699) de Fénelon.

37. À ce propos, voir l'article de J. Lecointe dans le présent volume et, du même auteur, «Lectures romanesques de Virgile à la Renaissance ", Cahiers de l'Association internationale des études françaises, 2001, n 53, p. 191-212.

38. P. de Deimier, L'Académie de l'art poétique, Paris, J. de Bordeaulx, 1610, p. 253 : «Que quelque temps apres, Lysimont par les charmes d'un faux rapport, reputant sa femme infidelle, se r'embarqueroit au desceu d'icelle, \& luy envoyeroit pour tout adieu \& confort une lettre, en laquelle il la taxeroit cruellement de luy avoir esté desloyale, \& luy attesteroit qu'il ne reviendroit jamais vers elle. »

39. O. S. de Claireville, Les Amours infidelles, Paris, A. de Sommaville, 1625. Ce petit roman se présente comme une narrativisation de trois héroïdes d'Ovide avec les «Amours de Paris et d'œEnone » et les « Amours de Jason et Médée ».

40. Ch. B. Beall note que, dans le roman du $\mathrm{XvII}^{\mathrm{e}}$ siècle, Virgile n'est le plus souvent imité que " dans la mesure où il a lui-même servi de modèle au Tasse » ("Note sur la Jérusalem délivrée et le Roman français », Revue de Littérature Comparée, XIXe année, 2, avril-juin 1939, p. 274-280 et ici p. 274).

\section{AUTHOR}

\section{ADRIENNE PETIT}

Université de Lille - EA 1061 (Analyses littéraires et histoire de la langue) 